\title{
Post Herpetic Neuropathy of Sinuvertebral Nerve: A Case Report
}

\author{
Seungyoun Kang ${ }^{1}$, Nitin Adsul ${ }^{1,2}$, Hyeun-Sung Kim ${ }^{1}$, Jongmin Sun ${ }^{1}$, Sungchan Chung ${ }^{1}$, Il-Tae Jang ${ }^{1}$ \\ ${ }^{1}$ Department of Neurosurgery, Nanoori Gangnam Hospital, Seoul, Korea; \\ ${ }^{2}$ Department of Spine Surgery, Sir Ganga Ram Hospital, New Delhi, India
}

Corresponding Author: Hyeun Sung Kim, MD, PhD Department of Neurosurgery, Nanoori Gangnam Hospital, 731, Eonju-ro, Gangnam-gu, Seoul 06048, Korea

Tel: $+82-2-6003-9767$

Fax: +82-2-3445-9755

E-mail: neuros@hanmail.net

Received: September 9, 2018 Revised: October 19, 2018 Accepted: October 29, 2018
Varicella-Zoster virus is a neurotropic virus of the herpes virus family that primarily affects sensory nerves. Herpes zoster causing sinuvertebral neuropathy has not been mentioned in the literature. A 55 years old man presented with low back pain, both buttocks, posterior thigh and leg pain over last 3 months. A straight leg raising test was positive on both sides. A left great toe dorsiflexion was decreased to $4 / 5$. The VAS score at admission for back and leg pain was $7 / 10$. The patient MRI was showing disc degeneration at L5-S1 level. We performed endoscopic interlaminar annuloplasty using radiofrequency ablation to denervate the sinuvertebral nerve attached to the annulus under epidural anesthesia. Patient symptoms completely relieved at the postoperative period and continued upto recent follow up of 6 months. The classical presentation of the patient after herpes zoster infection as back pain with referred leg pain, disc degeneration on MRI, intraoperative evidence of chronic neuropathy and almost complete improvement in patient symptoms after radiofrequency ablation makes it a first reported case of sinuvertebral neuropathy following herpes zoster infection.

Key Words: Post herpetic neuralgia, Sinuvertebral neuropathy, Radiofrequency ablation, Lumbar radiculopathy

\section{INTRODUCTION}

Varicella-Zoster virus is a neurotropic virus of the herpes virus family that primarily affects sensory nerves ${ }^{10)}$. We present here a case of a herpes zoster infection in 55 years old man causing sinuvertebral neuropathy. To the best of our knowledge, no article has described the association of herpes zoster infection with sinuvertebral neuropathy and its successful treatment using percutaneous endoscopic radiofrequency ablation using interlaminar approach.

\section{CASE REPORT}

A 55 years old man presented with low back pain, both buttocks, posterior thigh and leg pain over last 3 months. A straight leg raising test was positive on both sides. A left great toe dorsiflexion was decreased to $4 / 5$. The visual analog scale score at admission for back and leg pain was $7 / 10$. There was still hyperpigmentation of skin characteristics of old herpes zoster infection on the back, right buttock and thigh (Fig. 1). The patient was on antiviral medications when he initially developed
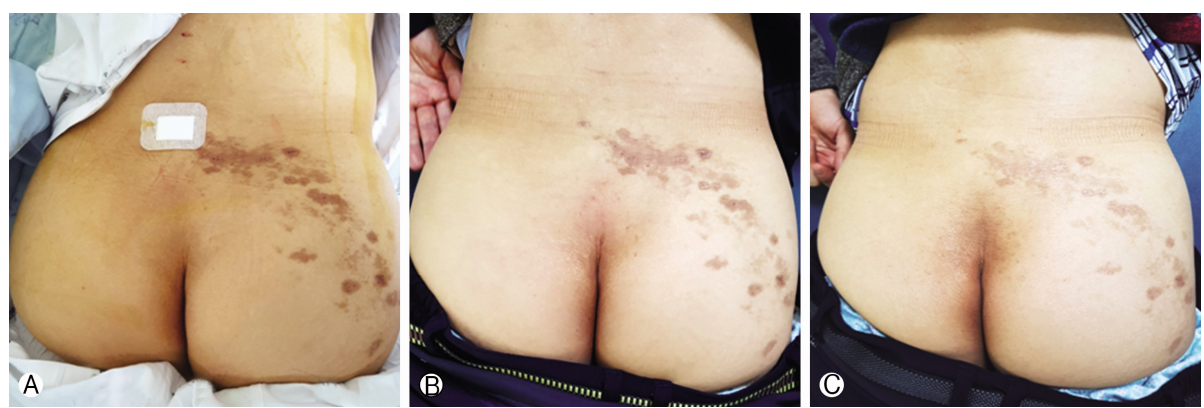

Fig. 1. Clinical picture showing the improvement in skin lesions over period of time (A). Immediate post-op (B) at one month follow up and (C) at three months follow up.

Copyright (C) 2018 The Korean Minimally Invasive Spine Surgery Society

This is an open access article distributed under the terms of the Creative Commons Attribution Non-Commercial License (http://creativecommons.org/licenses/by-nc/4.0/) which permits unrestricted non-commercial use, distribution, and reproduction in any medium, provided the original work is properly cited. 
the skin lesions before 3 months. Then he started on steroid and gabapentin but there was no improvement in back, buttock, thigh and leg pain. He also had the twice epidural neuroplasty but no improvement. The patient MRI was showing disc degeneration at L5-S1 level (Fig. 2A). Owing to severe pain refractory
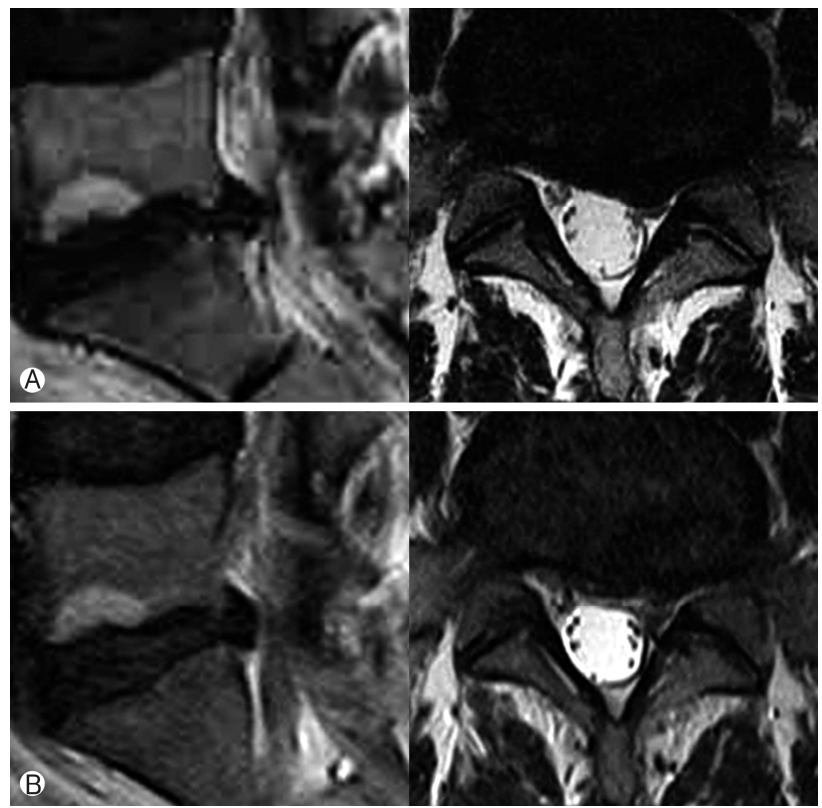

Fig. 2. (A) Preoperative T2-weighted MRI image of the L5-S1 level showing disc degeneration; (B) Postoperative MRI after annuloplasty.
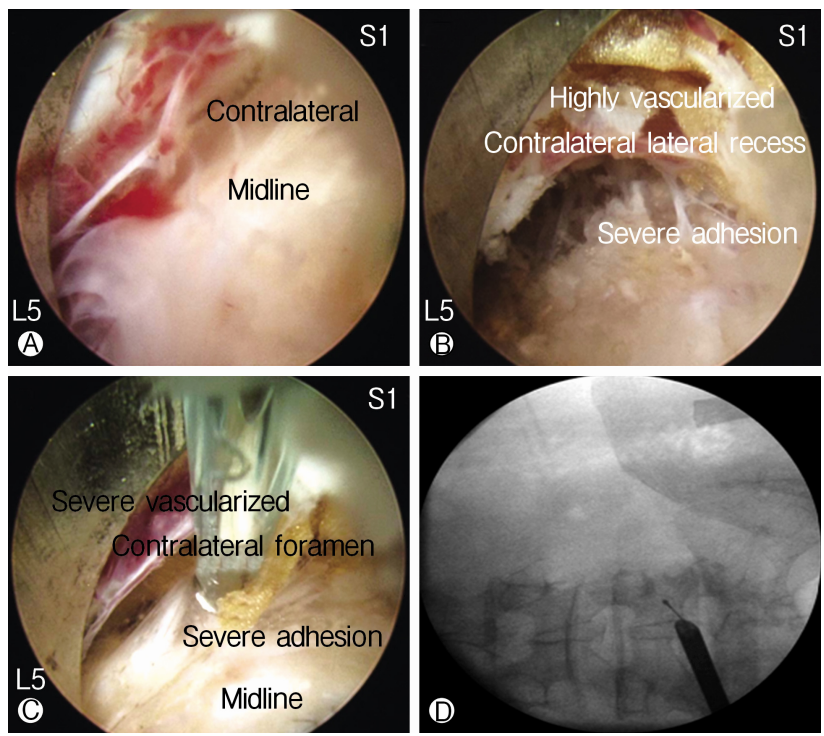

Fig. 3. Intraoperative images showing severe vascularization and adhesions in the midline $A$, a contralateral lateral recess (B) and contralateral foraminal area (C). C arm image (D) showing the position of the tip of the radiofrequency probe and working channel while doing adhesiolysis and annuloplasty as shown in image (C). to conservative management, we decided to do annuloplasty using structural preservation percutaneous endoscopic lumbar interlaminar approach ${ }^{13)}$ on the left side under epidural anaesthesia (Fig. 2B). We used an endoscope system with a 30-degree viewing angle, $7.3 \mathrm{~mm}$ outer diameter, and $4.7 \mathrm{~mm}$ working channel (Joimax $\mathrm{GmbH}$, Karlsruhe, Germany). The entire operation was performed under visual control and continuous irrigation with normal saline $(0.9 \%)$. There was severe vascularization in the midline and contralateral side (Fig. 3; Video 1) which was suggestive of sinuvertebral neuropathy ${ }^{11)}$. We performed annuloplasty using radiofrequency ablation (Ellman's bipolar radiofrequency electrocoagulator - Elliquence, Baldwin, New York, USA) to denervate the sinuvertebral nerve attached to the annulus. Radiofrequency ablation performed on the ipsilateral side over the contained disc and almost upto the contralateral foramen and dorsal root ganglion (Fig. 3). Patient symptoms completely relieved at the postoperative period and continued upto recent follow up of 6 months. The hyperpigmentation also improved significantly over the period of time (Fig. 1).

\section{DISCUSSION}

Herpes zoster is a viral disease occurs due to reactivation of the highly contagious varicella-zoster virus. During primary infection, the virus gains entry and persists in the sensory dorsal root ganglion. Reactivation of the varicella-zoster virus occurs due to depression of cell-mediated immunity and in aged patients ${ }^{16)}$. The thoracic dermatomes are affected in 50-56\%. The head area including trigeminal and other cranial nerves (VII and VIII) in $20 \%{ }^{7)}$. Rarely the virus affects cervical, lumbar and sacral segments ${ }^{6}$. The exact incidence of herpes zoster affecting the lumbar spine is not mentioned in the available literature.

Herpes zoster neuralgia should be considered in differential diagnosis of sciatica, as early treatment is of prime importance to avoid long-term neurological complications ${ }^{2,8}$. The treatment of herpes zoster includes pain management and antiviral medications. The neuralgic pain is treated in a stepwise manner using WHO analgesic ladder. The radiculopathy associated with herpes zoster can be treated with selective nerve root block or epidural neurolysis ${ }^{5,9,20)}$. An antiviral treatment should be administered within 48-72 hours after the onset of clinical features. It can

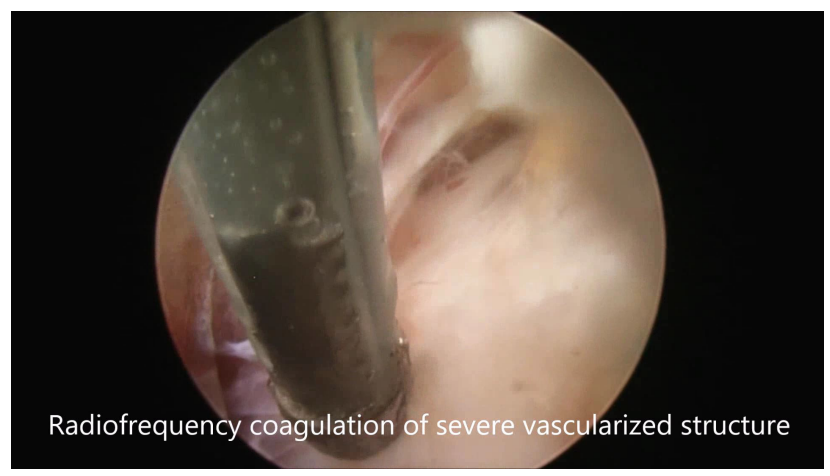

Video 1. Short Intraoperative video. 

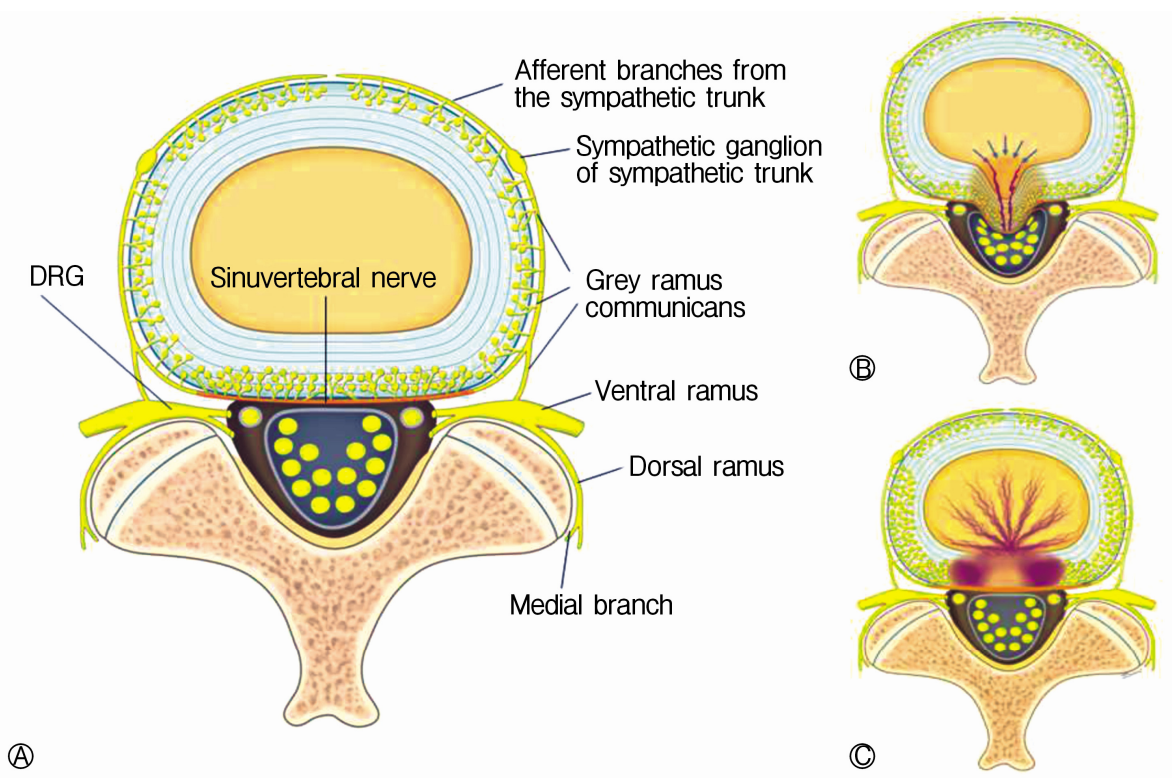

Fig. 4. Schematic diagram of an axial section of the spine showing. (A) Anatomy of the sinuvertebral nerve, normal state; (B) Disc degeneration with an annular tear; (C) Pathologic state where nerve grows inside the disc and gets sensitized around the posterior annulus and posterior longitudinal ligament.

be either oral or intravenous ${ }^{2)}$.

Indications for an antiviral therapy include:

- Age more than 50 years,

- Immunocompromised status,

- severe zoster of the trunk or extremities,

- zoster of the head, neck or sacral area, and

- severe dermatitis ${ }^{7)}$.

This antiviral drug needs to be given for a minimum of 7 days. Postherpetic neuralgia (PHN) is a chronic neuropathic pain after the herpes zoster $(\mathrm{HZ})$ infection and persists even after the cutaneous lesions have healed. Despite various treatment advances, many patients remain noncompliant to the current therapies and continue to have pain; physical and psychosomatic distress ${ }^{21}$. In our case, the patient received the complete course of antiviral drugs after he developed the dermatological manifestations. Due to persistent and painful sinuvertebral neuropathy, he visited us after 3 months of diagnosis of herpes zoster.

The herpes virus infection is also one of the factors, which promotes disc degeneration ${ }^{1)}$. Though the changes in lumbar spine morphology can be diagnosed using MRI, the precise location of the pain-inducing region cannot be distinguished ${ }^{14,17,19)}$.

Racz's also known as "epidural neuroplasty" is minimal invasive epidural catheter procedure. Its principle of action is local epidural lysis of adhesions, neurolysis of spinal nerve roots, and local lavage of proinflammatory mediators by repeated injections of local anesthetics, corticosteroids, hyaluronidase, and hypertonic saline solution ${ }^{22)}$. We performed this procedure twice but there was no improvement in pain which diminished the possibility of radiculopathy.

The sinuvertebral nerves arise in the intervertebral foramina
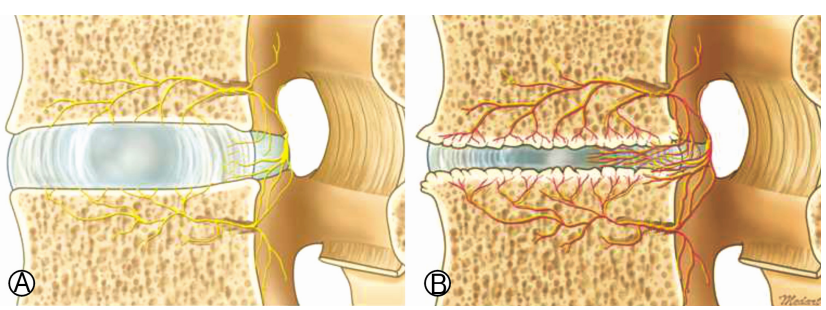

Fig. 5. Schematic diagram of sagittal section of spine showing. (A) Anatomy of the sinuvertebral nerve 's' and basivertebral nerve 'b' - normal state; (B) Pathologic state where nerve grows inside the disc and gets sensitized around the posterior annulus and posterior longitudinal ligament.

from 2 roots, 1 from the ventral primary ramus and the other from a ramus communicans (Fig. 4A) ${ }^{12)}$. These nerves are implicated in diffuse low back pain because of their pathway and its sympathetic component. Pain impulses coming from the intervertebral disk, posterior longitudinal ligament, and ventral part of the dura join the L2 spinal ganglion (somatic system) via the rami communicantes and sympathetic trunk. Therefore the sinuvertebral nerve as a carrier of deep somatic pain is related to referred pain and exhibits similar pain characteristics as radicular pain. Chronic derangement of the sinuvertebral nerve can induce neural degeneration, infiltration of neural tissue into the intradiscal space, and aggravation of disk degeneration (Figs. 4 and 5) ${ }^{18)}$.

The endoscopic ablation of sinuvertebral nerve resulted in almost complete improvement in patient symptoms. The endoscopic interlaminar approach is the most minimally invasive ap- 
proach to diagnose and treat sinuvertebral neuropathy for the L5-S1 level. Transforaminal access is difficult for L5-S1 level since, the highest part of the iliac crest is located above the L5-S1 level, making it difficult to access the L5-S1 disk space ${ }^{3,4,15)}$. In addition, the L5 transverse process is the largest among the lower vertebral bodies and bends downward ${ }^{12)}$. The sacral ala is also responsible for narrowing the L5-S1 intervertebral foramen.

The classical presentation of the patient after herpes zoster infection as back pain with referred leg pain, disc degeneration on MRI, intraoperative evidence of chronic neuropathy and almost complete improvement in patient symptoms after radiofrequency ablation makes it a first reported case of sinuvertebral neuropathy following herpes zoster infection.

\section{CONCLUSION}

The possibility of sinuvertebral neuropathy must be kept in mind as a sequel to herpes zoster, which mimics as lumbar radiculopathy. A percutaneous endoscopy is a powerful tool in diagnosis and management of this rare scenario. Early diagnosis and treatment of this condition are of the essence to improve outcome.

\section{Financial Disclosure}

No funds were received in support of this work.

No benefits in any form have been or will be received from a commercial party related directly or indirectly to the subject of this manuscript.

\section{REFERENCES}

1. Alpantaki K, Katonis P, Hadjipavlou A, Spandidos D, Sourvinos G: Herpes virus infection can cause intervertebral disc degeneration: a causal relationship? J Bone Joint Surg Br 93:1253-1258, 2011

2. Bader MS: Herpes zoster: diagnostic, therapeutic, and preventive approaches. Postgraduate Medicine 125:78-91, 2013

3. Chen HT, Tsai CH, Chao SC, Kao TH, Chen YJ, Hsu HC, et al: Endoscopic discectomy of L5-S1 disc herniation via an interlaminar approach: Prospective controlled study under local and general anesthesia. Surg Neurol Int 2:93, 2011

4. Chumnanvej S, Kesornsak W, Sarnvivad P, Paiboonsirijit S, Kuansongthum V: Full endoscopic lumbar discectomy via interlaminar approach: 2-year results in Ramathibodi Hospital. J Med Assoc Thai 94:1465-1470, 2011

5. Conliffe TD, Dholakia M, Broyer Z: Herpes zoster radiculopathy treated with fluoroscopically-guided selective nerve root injection. Pain Physician 12:851-853, 2009

6. Gross G: Clinical picture and complications of herpes zoster: the view of the dermatologist: Herpes Zoster: Karger Publishers, pp20-36, 2006

7. Gross G, Schöfer H, Wassilew S, Friese Ke, Timm A, Guthoff $\mathrm{R}$, et al: Herpes zoster guideline1 of the german dermatology society (DDG). Journal of clinical virology 26:277-289, 2003

8. Hackenberg RK, von den Driesch A, König DP: Lower back pain with sciatic disorder following L5 dermatome caused by herpes zoster infection. Orthopedic Reviews 7, 2015

9. Hardy D: Relief of pain in acute herpes zoster by nerve blocks and possible prevention of post-herpetic neuralgia. Canadian Journal of Anesthesia 52:186-190, 2005

10. Helfgott SM, Picard DA, Cook JS: Herpes zoster radiculopathy. Spine 18:2523-2524, 1993

11. Ito K, Creemers L: Mechanisms of intervertebral disk degeneration/injury and pain: a review. Global Spine Journal 3:145-151, 2013

12. Kim HS, Paudel B, Chung SK, Jang JS, Oh SH, Jang IT: Transforaminal epiduroscopic laser ablation of sinuvertebral nerve in patients with chronic diskogenic back pain: technical note and preliminary result. Journal of Neurological Surgery Part A: Central European Neurosurgery 78:529-534, 2017

13. Lee J-S, Kim H-S, Jang J-S, Jang I-T: Structural preservation percutaneous endoscopic lumbar interlaminar discectomy for L5-S1 herniated nucleus pulposus. BioMed research International 2016, 2016

14. Majumdar S, Link TM, Steinbach LS, Hu S, Kurhanewicz J: Diagnostic tools and imaging methods in intervertebral disk degeneration. Orthopedic Clinics 42:501-511, 2011

15. Moliterno JA, Knopman J, Parikh K, Cohan JN, Huang QD, Aaker GD, et al: Results and risk factors for recurrence following single-level tubular lumbar microdiscectomy. J Neurosurg Spine 12:680-686, 2010

16. Nagel MA, Gilden D: Neurological complications of VZV reactivation. Current Opinion in Neurology 27:356, 2014

17. Pfirrmann CW, Metzdorf A, Zanetti M, Hodler J, Boos N: Magnetic resonance classification of lumbar intervertebral disc degeneration. Spine 26:1873-1878, 2001

18. Raoul S, Faure A, Robert R, Rogez J-M, Hamel O, Cuillère $\mathrm{P}$, et al: Role of the sinu-vertebral nerve in low back pain and anatomical basis of therapeutic implications. Surgical and Radiologic Anatomy 24:366-370, 2002

19. Sąsiadek MJ, Bladowska J: Imaging of degenerative spine disease the state of the art. Adv Clin Exp Med 21:133-142, 2012

20. Shakir A, Kimbrough DA, Mehta B: Postherpetic neuralgia involving the right C5 dermatome treated with a cervical trans foraminal epidural steroid injection: a case report. Archives of Physical Medicine and Rehabilitation 88:255-258, 2007

21. Singh S, Gupta R, Kaur S, Kaur J: Post-herpetic neuralgia: A review of current management strategies. Indian Journal of Pain 27:12, 2013

22. Wagner K, Sprenger T, Pecho C, Kochs E, Tölle T, Berthele A, et al: Risks and complications of epidural neurolysis--a review with case report. Anasthesiologie, Intensivmedizin, Notfallmedizin, Schmerztherapie: AINS 41:213-222, 2006 